\title{
Photovoltaic OLED Driver for Low-Power Stand-Alone Light-to-Light Systems
}

\author{
Rasmus Overgaard Ploug ${ }^{1}$, Arnold Knott ${ }^{1}$ \\ ${ }^{I}$ Department of Electrical Engineering, Technical University of Denmark, \\ Kgs. Lyngby, Denmark \\ roploug@elektro.dtu.dk
}

\begin{abstract}
Photovoltaic (PV) stand-alone systems need to achieve multiple energy conversion modes. I.e. the energy conversion from PV to a local energy storage as well as energy conversion from the energy storage to the load. This paper documents the practical design considerations for the development of a three-port-converter for this purpose optimized for the specifications for driving an Organic Light Emitting Diode (OLED) panel intended for lighting purposes. By using a three-port-converter, featuring shared components for each conversion mode, the converter reaches $97 \%$ efficiency at $1.8 \mathrm{~W}$ during conversion from photovoltaic panel to the battery, and $97 \%$ in the area $1.4 \mathrm{~W}$ to $2 \mathrm{~W}$ for power delivery to the OLED.
\end{abstract}

Index Terms-Power electronics; photovoltaic systems; organic light emitting diodes; energy efficiency.

\section{INTRODUCTION}

The recent years small Photovoltaic (PV) driven standalone battery systems for urban lighting has seen more and more interest. Not only because of the green energy aspect, but also because of immense cost savings by not having to connect these lighting systems to the electrical grid and to route electrical power cables through the ground to the lighting device. As a stand-alone system, such a product should be able to harvest energy from the solar irradiation, convert and store this energy in a local battery and, during the night, convert the energy necessary to illuminate its lighting fixture with the battery power. On system level this is also denoted as a Light-to-Light (LtL) system [1], [2]. In order to obtain the correct energy conversions, power electronics has to be designed with highest degree of efficiency to relax the oversize requirements of the PV panel and the battery respectively.

Research has been going on on the power electronics design for this product type in [1]-[6]. However, these works are based on driving conventional Light Emitting Diodes (LED) with the voltage and current levels associated with them. With the emerging introduction of Organic Light Emitting Diodes (OLED) to the market, new lighting concepts can be developed to suit the urban environment lighting demands, and hereby the necessity of developing the electronic power converter optimized for OLED specifications.

Manuscript received 21 October, 2015; accepted 28 March, 2016.

This research was funded by ELFORSK - the research and development program of the Danish Energy Association.

\section{ORGANIC LIGHT EMITTING DIODES}

OLEDs for lighting purposes are flat and thin organic layers applied to a substrate between an anode and a cathode. Usually an OLED lighting panel is made of organic layers capable of producing red, blue and green light. They can be put on top of each other to produce the impression of 'white' light with excellent color rendering capabilities [7]. As such, an OLED panel gives the impression of an illuminated 'surface' rather than lighting point-sources from LEDs. The light emission of OLED panels is theoretically proportional to the amount of electrons flowing through the light-emitting layers hence it is said to be proportional to the current applied [8]. Furthermore, studies of the ageing process of OLED panels have shown that the static resistance of the OLEDs increase with ageing [9]. In other words: The voltage needed for the same current/illumination increases over time, hence, to ensure a somewhat consistent illumination of an OLED panel during its lifetime, the OLED panel should be driven by a constant current controlled source.

TABLE I. EXAMPLES OF OPERATING POINTS OF SELECTED PANELS FROM LG PORTFOLIO OF OLED LIGHTING PANELS [10].

\begin{tabular}{|c|c|c|c|c|}
\hline Panel size & Current & Voltage & Power & $\begin{array}{c}\text { Luminous } \\
\text { flux }\end{array}$ \\
\hline $100 \times 100 \mathrm{~mm}$ & $150 \mathrm{~mA}$ & $8.5 \mathrm{~V}$ & $1.275 \mathrm{~W}$ & $75 \mathrm{~lm}$ \\
\hline $200 \times 50 \mathrm{~mm}$ & $150 \mathrm{~mA}$ & $8.5 \mathrm{~V}$ & $1.275 \mathrm{~W}$ & $75 \mathrm{~lm}$ \\
\hline $140 \times 140 \mathrm{~mm}$ & $300 \mathrm{~mA}$ & $8.5 \mathrm{~V}$ & $2.55 \mathrm{~W}$ & $150 \mathrm{~lm}$ \\
\hline $320 \times 110 \mathrm{~mm}$ & $500 \mathrm{~mA}$ & $8.5 \mathrm{~V}$ & $4.25 \mathrm{~W}$ & $250 \mathrm{~lm}$ \\
\hline
\end{tabular}

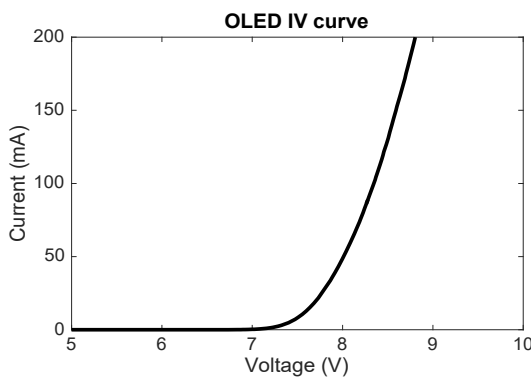

Fig. 1. Measured IV-curve of LG N6BA30C OLED lighting panel. $200 \mathrm{x}$ $50 \mathrm{~mm}$.

Usually an OLED panel is specified for a specific operating point. In Table I some specifications from the OLED portfolio of the manufacturer LG are presented. Furthermore, an OLED is (as the name implies) electrically a diode as can be seen from the measured IV-curve of the LG N6BA30C OLED panel shown in Fig. 1. 


\section{SYSTEM DESIGN}

An electronic converter for outdoor light-to-light purposes should be able to work in two modes:

1. In the daytime: Convert energy from the PV panel while maintaining Maximum Power Point Tracking (MPPT) in order to harvest as much energy from the solar irradiation as possible.

2. In the night: Convert stored energy from the battery to the operating point of the OLED panel.

Hence this application calls for a Three-Port-Converter (TPC) with two Single-Input Single-Output (SISO) operating modes as sketched in Fig. 2 [10]-[13].

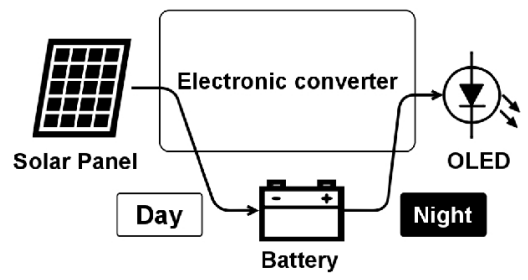

Fig. 2. System design of the converter.

For this study the selected PV panel consists of three panels in parallel each having the specifications listed in Table II.

TABLE II. PV SPECIFICATIONS

\begin{tabular}{|c|c|}
\hline$V_{m p p}$ & $9 \mathrm{~V}$ \\
\hline$I_{m p p}$ & $150 \mathrm{~mA}$ \\
\hline$P_{m p}$ & $1.35 \mathrm{~W}$ \\
\hline$I_{s c}$ & $165 \mathrm{~mA}$ \\
\hline$V_{o c}$ & $9.9 \mathrm{~V}$ \\
\hline
\end{tabular}

The battery is a Li-ion battery with 3 cells in parallel obtaining 4.5 Ah at 3.6 V and the OLED is assumed to have the same operating voltage as the LG N6BA30C, but the converter should be able to drive up to $500 \mathrm{~mA}$ in order to facilitate various OLED panel sizes. However, efficiency wise, a current target of $150 \mathrm{~mA}$ is chosen.

\section{A. Topology}

Since the voltage from the PV panel has to be stepped down to the battery voltage in the daytime and the battery voltage has to be stepped up in the nighttime, a combined buck and boost converter is suggested as topology for the three-port-converter. This can be seen in Fig. 3 .

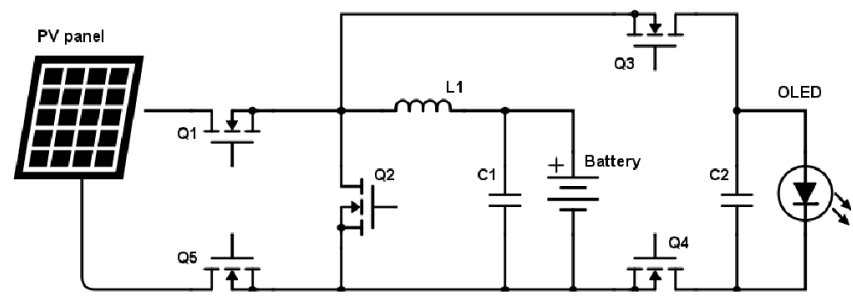

Fig. 3. Converter topology. Q4 and Q5 are power flow control switches.

At daytime, when charging the battery, the converter operates in buck-mode with Q1 as control switch and Q2 as synchronous rectifier (see Fig. 4). At nighttime, when delivering power to the OLED, the converter operates in boost-mode now with Q2 as the control switch and Q3 as the synchronous rectifier (see Fig. 5). Q4 and Q5 are power flow control switches. Q4 prevents unwanted current to flow in the OLED when in buck-mode as the body diode in Q3 can become forward biased. Q5 prevents unwanted reverse current in the PV panel during boost-mode. Q5 could alternatively be exchanged with a diode to block current from flowing back to the PV panel, however, a controlled switch is highly preferred since the voltage drop caused by the ON-resistance from a switch is much lower than the voltage drop from a diode especially at low currents. Q4 and Q5 are always on or always off depending on the operating mode, hence switching losses can be neglected for these switches. The benefit of using this topology compared to a solution comprising of a buck and a boost is evidently the sharing of the inductor and Q2 switch between the two operating modes.

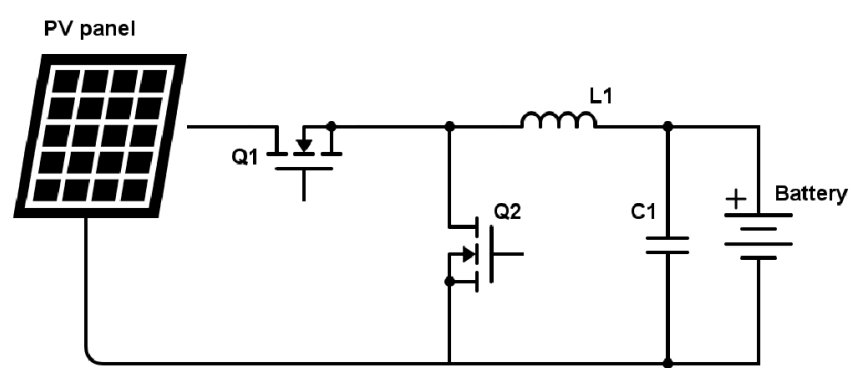

Fig. 4. Converter operating in buck-mode. Q5 is omitted since this is always 'on' when operating in buck-mode.

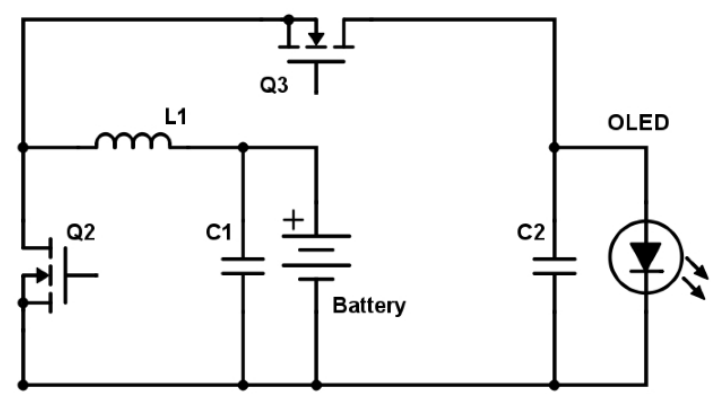

Fig. 5. Converter operating in boost-mode. Q4 is omitted since it is always 'on' when operating in boost-mode.

A similar topology has been documented for a PV-LED system in [2]. However, since a higher LED voltage was needed the step-up converter in that work was configured as a tapped-boost converter.

\section{EFFICIENCY AND COMPONENT SELECTION}

Solar irradiation changes continuously during the daytime. Clouds are often covering, or partly covering, the sun reducing the irradiation at ground level and thus reducing the output power from the PV panel. Therefore, for a standalone PV system, the size of the PV panel and/or the battery has to be cleverly selected with a degree of oversize margin in order to ensure consistent functionality throughout the year with varying irradiation and lengths of daytime. Finding this tradeoff is outside the scope of this work. However, the converter efficiency is very important in utilizing as much energy as possible from the sun, relaxing the oversize tradeoff requirements of the rest of the components in the system. In [14] it was emphasized that especially the system efficiency in stand-alone PV-systems at low power levels has to be maximized based on solar irradiation data from a northern latitude. Furthermore, in the same work, a 
semiconductor loss evaluation was done concluding that the gate energy loss is the dominant part of the losses, hence the MOSFETs should be selected especially based on having a low gate charge requirement.

In addition, all utility components for the converter (e.g. Operational amplifiers, voltage converters, gate drivers, sense resistors etc.) has been selected especially based on their power consumption to increase the overall system efficiency. The selection of the dominant components and their relation to the converter efficiency is presented below:

\section{A. MOSFETS}

Based on [14] the switching devices was all chosen to be the MOSFET BSC050NE2LS from Infineon featuring the key parameters seen in Table III (from datasheet [15]).

TABLE III. BSC050NE2LS KEY PARAMETERS.

\begin{tabular}{|c|c|}
\hline$V_{D S}$ & $25 \mathrm{~V}$ \\
\hline$R_{D S}(o n)$ & $5.7 \mathrm{~m} \Omega$ \\
\hline$Q_{g}$ & $5.0 \mathrm{nC}$ \\
\hline
\end{tabular}

\section{B. Controller}

In order to facilitate the Maximum Power Point Tracking (MPPT) of the PV panel while monitoring the battery charge status a digital control scheme based on the perturb and observe' method was selected and implemented on an ultra low power Texas Instruments MSP430F5172 microcontroller. This microcontroller features a high resolution timer module, which was used to output PWM signals for the power switches at $104 \mathrm{kHz}$ with an 11 Bit resolution. For the boost converter control it was set up for constant current control to interface with OLEDs.

\section{C.Inductor}

Since the converter switching frequency is relatively low $(104 \mathrm{kHz})$, the DC-resistance of the inductor was identified as the main inductor loss component. Thus a Murata 60B104C $100 \mu \mathrm{H}$ inductor was chosen to obtain highest possible efficiency. It is rated to an RMS current at $7.5 \mathrm{~A}$ and is as such oversized for the current levels of this application. However, with a DC Resistance of $\max 25 \mathrm{~m} \Omega$ it should keep inductor DC-losses at a minimum.

\section{D.Dead Time Selection}

In order to prevent shoot through in the switching legs of a converter dead time is usually inserted in the PWM control signals for the MOSFETs. In this work a simple dead time circuit as seen in Fig. 6 is used. It contains an RC low pass filter to delay the rising flank of the control signal inserted before the gate drivers. A schottky diode is inserted to quickly discharge the gate driver input. This circuit is applied to the control signals for Q1, Q2 and Q3.

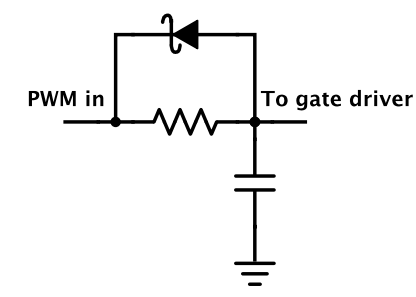

Fig. 6. Dead time generating circuit.

By increasing these dead times, the switching loss of the synchronous rectifier can be considerably reduced due to Zero Voltage Switching (ZVS) or partial ZVS in a chosen operating point. However, if too long a dead time is introduced the conduction loss of the intrinsic body diode begins to dominate hence a tradeoff hereof exists. In Fig. 7 and Fig. 8 the body diode conduction impact on the switching signals is shown during too long dead times. For the boost converter (Fig. 7) it can be seen that control FET dead time $\left(\mathrm{t}_{\mathrm{dt} \text { on }}\right)$ always should be kept as short as possible since body diode conduction occurs as soon as the synchronous rectifier is switched off. However, for the synchronous rectifier dead time ( $\left.t_{\text {dt_off }}\right)$ the switch voltage (Vsw) commutates to the output voltage before body diode conduction commences. Hence the optimal $t_{d t}$ off should make the synchronous rectifier turn on just before the body diode begins to conduct.

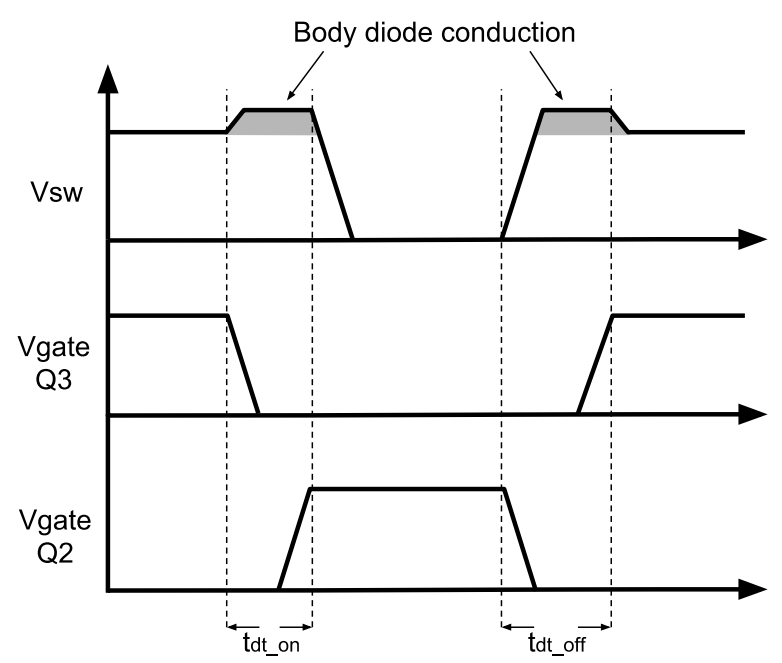

Fig. 7. Exaggerated dead time effect on boost converter waveforms. The intrinsic body diode of the MOSFETs conduct during too long dead times. $t_{d t}$ on denotes control FET dead time and $t_{d t}$ off denotes synchronous rectifier dead time.

The same principle applies to the buck converter waveforms (Fig. 8) namely that $t_{d t}$ on should be kept as short as possible and $t_{d t \text { off }}$ should be optimized for the commutating voltage switching the synchronous rectifier on just before body diode conduction.

In this topology the synchronous rectifier of the buck (Q2) is the same as the control switch of the boost part and thus they are sharing their dead time circuit. This creates a contradiction since, for the boost part, the dead time should be as short as possible and for the buck part it should have a certain length in order to achieve ZVS. Hence an optimal length of dead time for Q2 must be found in order to make a compromise between partly ZVS of the buck synchronous rectifier and body diode conduction of the boost control FET.

It was chosen to optimize efficiency from dead time for $0.5 \mathrm{~W}$ for the buck part to obtain good results in the lowest power area and to optimize for $1.275 \mathrm{~W}$ for the boost part corresponding to the operating point of the select OLED panel. To find the optimal dead time for Q2 the switch-on energy loss for the buck synchronous rectifier was estimated as well as the boost body diode energy loss. Switch-on loss was estimated based on datasheet values using the method from [16] giving the switch-on energy loss as 


$$
W_{\text {on }}=V_{\text {bus }} I_{\text {on }} \frac{t_{\text {irise }}+t_{\text {vfall }}}{2},
$$

where $V_{\text {bus }}$ is the voltage across the MOSFET at the switch event which can be calculated from the load current, the deadtime $t_{Q 2 d t}$ and the output capacitance of the MOSFETs (see appendix A), $I_{o n}$ is the current, $t_{\text {irise }}$ is the current rise time from the datasheet and $t_{v f a l l}$ is the voltage fall time calculated from the datasheet (see appendix B).



Fig. 8. Exaggerated dead time effect on buck converter waveforms, the intrinsic body diode of the MOSFETs conduct during long dead times. $t_{d t \_}$on denotes control FET dead time and $t_{d t \_}$off denotes synchronous rectifier dead time.

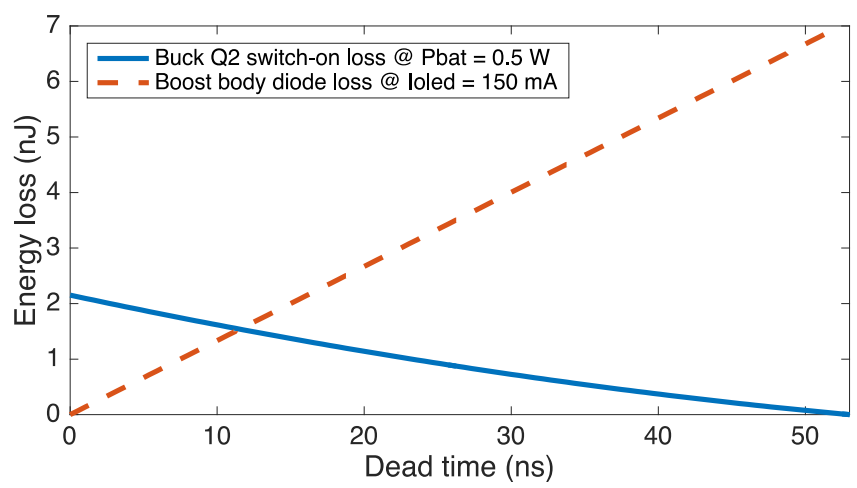

Fig. 9. Buck Q2 switch-on energy loss@0.5 W compared to boost Q2 body diode energy loss per switching event when outputting $150 \mathrm{~mA}$ to the OLED.

Body diode conduction loss from the boost control FET was estimated to be

$$
W_{\text {bodydiode }}=t_{Q 2 d t} I_{O L E D} V_{S D}
$$

where $t_{Q 2 d t}$ is the Q2 dead time, $I_{O L E D}$ is the output current to the OLED and $V_{S D}$ is the intrinsic body diode voltage drop of Q2. The losses are plotted in Fig. 9 where it can be seen that the lines intersect at a dead time around $11 \mathrm{~ns}$ deeming that to be a fair compromise.

\section{V.RESULTS}

The converter was realized as can be seen in Fig. 10 (top view). The calculated dead time for Q2 was implemented using the circuit in Fig. 6, and tuned so a 10 ns body diode conduction was visible in the boost converter switching waveform turn-on flank at the chosen operating point, as seen in Fig. 11.

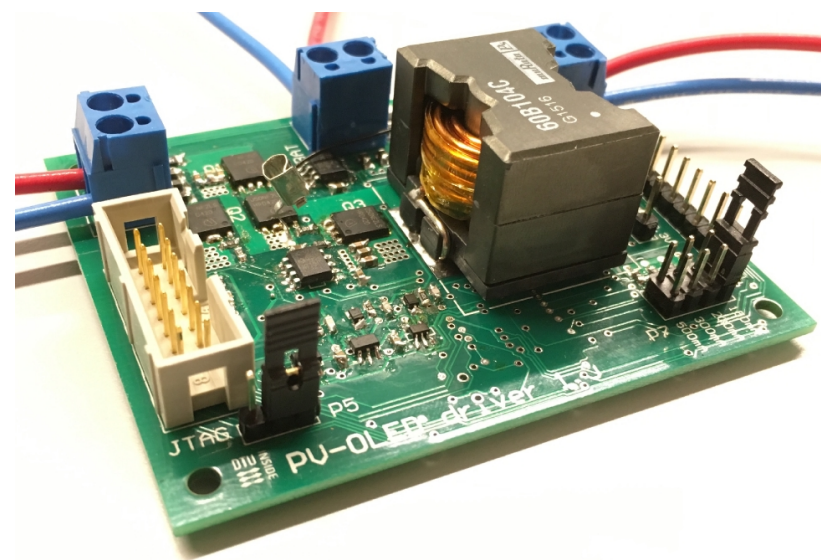

Fig. 10. Top-view of prototype. The prototype was realized on a 2-layer PCB.

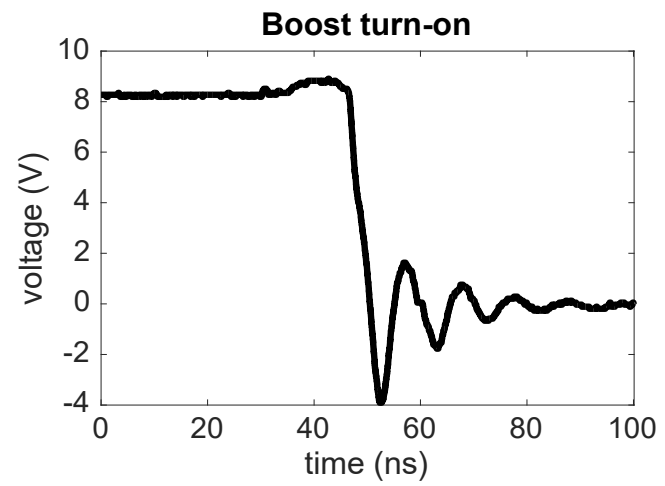

Fig. 11. Switch node waveform at the boost converter at $1.3 \mathrm{~W}$. Body diode conduction is visible as $10 \mathrm{~ns}$ voltage rise between the turn-off of Q2 and the turn-on of Q3.

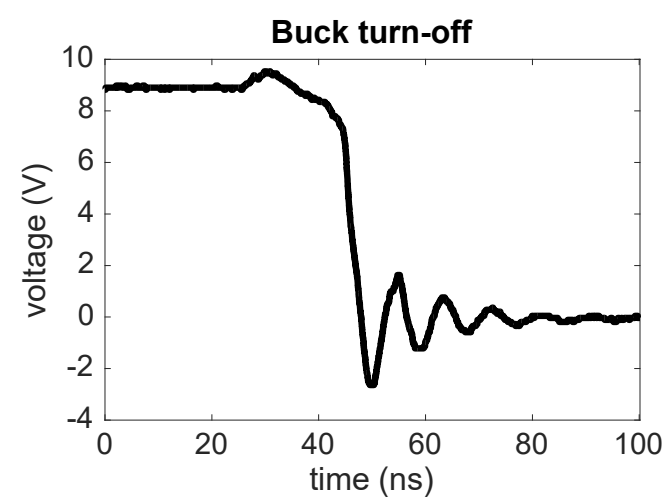

Fig. 12. Switch node voltage waveform at $0.5 \mathrm{~W}$ buck-mode turn-off with same dead time as in Fig. 11. It can be seen that during the dead time the switch node voltage manages to fall from $9 \mathrm{~V}$ to approximately $7.5 \mathrm{~V}$ before the synchronous rectifier Q2 turns-on.

In addition, also the turn-off flank of the boost converter is included as seen in Fig. 13. With no sharing dead time circuit of the boost converter synchronous rectifier, the switch node voltage can be allowed to fully commutate to get ZVS turn-on of Q3 at the chosen operating point with a dead time of approximately $40 \mathrm{~ns}$.

The resulting efficiency of the converter was measured with high precision Agilent 34410A multimeters synchronized with computer software to make simultaneous and automatic measurements. The efficiency measurements are made measuring the input and output power of the whole converter, i.e. all power usage for microcontroller, op-amps, logic, circuits etc. are included in the graphs. For the buck- 
conversion the efficiency at the $0.5 \mathrm{~W}$ target was measured to $94 \%$ with a peak at $97 \%$ when operating at $1.8 \mathrm{~W}$. The curve is plotted in Fig. 14.

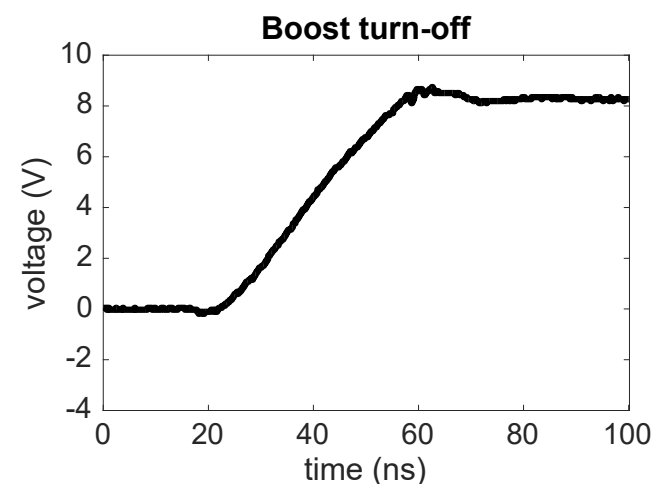

Fig. 13. Switch node voltage at the boost converter turn-off event. Dead time is tuned so that the voltage fully commutates before the synchronous rectifier Q3 is turned on.

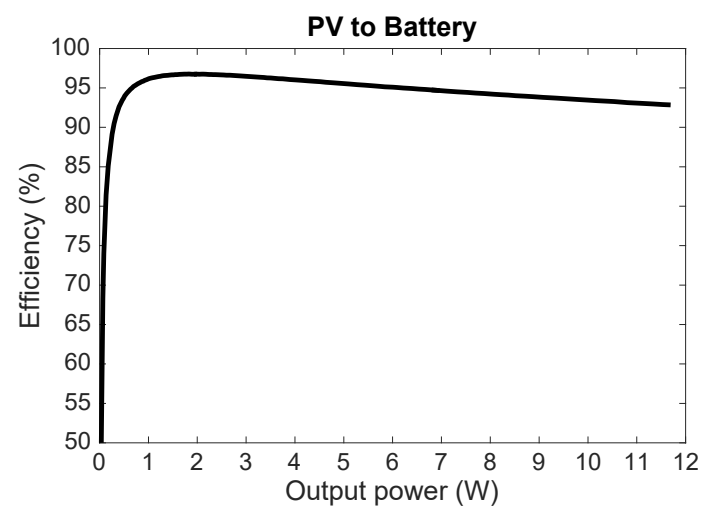

Fig. 14. Efficiency curve of the converter operating in buck-mode. It can be seen that the converter excels in having its highest efficiency at the lowest power obtaining $94 \%$ at $0.5 \mathrm{~W}$ and peaking at $97 \%$ at $1.8 \mathrm{~W}$

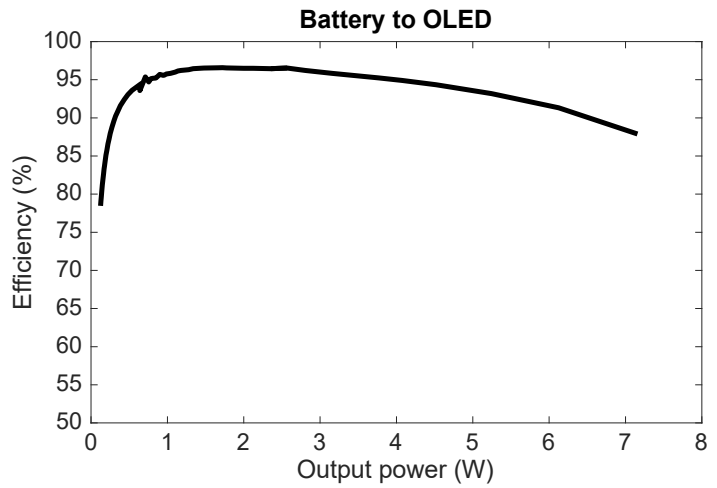

Fig. 15. Efficiency curve of the converter operating in boost mode. The converter reaches $96 \%$ efficiency at the $1.275 \mathrm{~W}$ target approaching $97 \%$ in the $1.4 \mathrm{~W}-2 \mathrm{~W}$ output power range.

For the boost-conversion, when transferring energy from the battery to the OLED panel, the converter reaches $96 \%$ at the $1.275 \mathrm{~W}$ operating point approaching $97 \%$ in the $1.4 \mathrm{~W}-2 \mathrm{~W}$ output power range as can be seen at the efficiency curve plotted in Fig. 15.

\section{CONCLUSIONS}

This paper presented a solution for a three-port converter intended for driving OLED lighting from a battery in a boost configuration, and charging the battery from a PV-panel in a buck configuration with MPPT, making the whole system a Light-to-Light system. The used topology featured reuse of one of the switches and the power inductor between the two operating modes. The converter was set to be optimized for the very low power area around $0.5 \mathrm{~W}$ when bucking, and for $1.275 \mathrm{~W}$ at the OLED side corresponding to the operating point of a select OLED panel. Measured efficiency showed $94 \%$ at $0.5 \mathrm{~W}$ when bucking with a peak of $97 \%$ at $1.8 \mathrm{~W}$, and $96 \%$ at $1.3 \mathrm{~W}$ when boosting with a peak of $97 \%$ in the $1.4 \mathrm{~W}-2 \mathrm{~W}$ output power area. Proving that this topology is capable of driving OLED panels in LtL configurations at these power levels while using only one power inductor. Future work into optimizing the converter efficiency is identified as investigating alternative dead time methods for this converter type either by separating the dead time circuits for Q2 or investigating adaptive dead time methods suitable for this converter type.

\section{APPENDIX A}

From [16]: The instantaneous switch-node voltage across the Q2 during buck-mode, $V_{b u s}$, can be calculated from the inductor peak-to-peak ripple current given by

$$
\Delta i=\frac{\left(\mathrm{V}_{P V}-\mathrm{V}_{B a t}\right) \mathrm{D}}{f_{s w} L} .
$$

The instantenous turn-off current at when Q1 turns off is then

$$
I_{\text {turn-off }}=I_{b a t}+\frac{\Delta i}{2}
$$

Then, before the synchronous rectifier Q2 turns on, the switch node voltage falls to zero depending on the output charge as

$$
t_{\text {fall }}=\frac{Q_{O S S}}{I_{\text {turn-off }}},
$$

where $Q_{\text {OSS }}$ is the total output charge of both Q1 and Q2 . From hereoff the negative slope of the switch node voltage can be calculated and $V_{b u s}$ estimated to a be dependent on the Q2 deadtime $t_{Q 2 d t}$

$$
V_{b u s}=V_{P V}-\frac{V_{P V}}{t_{\text {fall }}} t_{t Q 2 d t} .
$$

\section{APPENDIX B}

Using the method from [16] the voltage fall time $t_{v f a l l}$ can be estimated by

$$
t_{v f a l l}=\frac{t_{v f a l l 1}+t_{v f a l l 2}}{2},
$$

where:

$$
\begin{aligned}
& t_{v f a l l 1}=V_{b u s} R_{G} \frac{C_{G D 1}}{V_{d r}-V_{P L}}, \\
& t_{v f a l l 2}=V_{b u s} R_{G} \frac{C_{G D 2}}{V_{d r}-V_{P L}},
\end{aligned}
$$


here $R_{G}$ is gate-resistance, $V_{d r}$ is gate drive voltage, $V_{P L}$ is gate-plateau voltage and $C_{G D 1}$ and $C_{G D 2}$ is the gate capacitances associated with switching obtainable from the MOSFET datasheet. Note that the voltage drop regarding to the MOSFET on-resistance has been neglected.

\section{REFERENCES}

[1] N. Femia, M. Fortunato, M. Vitelli, "Light-to-light: PV-fed LED lighting systems", IEEE Trans. Power Electronics, vol. 28, no. 8, 2013. [Online]. Available: http://dx.doi.org/10.1109/TPEL.2012. 2229297

[2] M. C. Mira, A. Knott, M. A. E. Andersen, "Three-port converter for low-power stand-alone light-to-light systems (submitted for publication)", IEEE Energy Conversion Congress \& Expo 2016.

[3] S. Duryea, S. Islam, W. Lawrance, "A battery management system for stand alone photovoltaic energy systems", IEEE Industry Applications Magazine, 2001. [Online]. Available: http://dx.doi.org/ 10.1109/2943.922452

[4] J. Antonio Barros Vieira, A. Manuel Mota, "Implementation of a stand-alone photovoltaic lighting system with MPPT battery charging and LED current control", IEEE Int. Conf. Control Applications (CCA), 2010. [Online]. Available: http://dx.doi.org/10.1109/CCA 2010.5611257

[5] M. C. Mira, A. Knott, O. C. Thomsen, M. A. E. Andersen, "Boost converter with combined control loop for a stand-alone photovoltaic battery charge system", IEEE Control and Modeling for Power Electronics (COMPEL), 2013. Available: http://dx.doi.org/10.1109/ COMPEL.2013.6626428

[6] M. C. Mira, A. Knott, M. A. E. Andersen, "Analysis and comparison of magnetic structures in a tapped boost converter for LED applications", IEEE Electronics and Application Conf. Exposition (PEAC), 2014. [Online]. Available: http://dx.doi.org/ 10.1109/PEAC. 2014.7037923

[7] Yi-Lu Chang, Zheng-Hong Lu, "White organic light-emitting diodes for solid-state lighting", IEEE Journal of Display Technology, vol. 9 no. 6, 2013. [Online]. Available: http://dx.doi.org/10.1109/JDT.
2013.2248698

[8] Tokihisa Kawabata, Yoshi Ohno, "Optical measurements of OLED panels for lighting applications", Journal of Modern Optics, 2013. [Online]. Available: http://dx.doi.org/10.1080/09500340.2013. 806681

[9] P. Dupuis, A. Alchaddoud, L. Canale, G. Zissis, "OLED ageing signature characterization under combined thermal and electrical stresses", IEEE Int. Symposium on Electrical Insulating Materials ISEIM 2014. [Online]. Available: http://dx.doi.org/10.1109/ISEIM. 2014.6870781

[10] LG Display Products. [Online]. Available: http://www.lgoledlight.com

[11] Neng Zhang, Danny Sutanto, Kashem M. Muttaqi, "A review of topologies of three-port DC-DC convertersfor the integration of renewable energy and energy storage system", Elsevier Ltd Renewable and Sustainable Energy Reviews, vol. 56, 2016. [Online]. Available: http://dx.doi.org/ 10.1016/j.rser.2015.11.079

[12] M. C. Mira, A. Knott, M. A. E. Andersen, "A three-port topology comparison for a low power stand-alone photovoltaic system", IEEE Int. Power Electronics Conf. (IPEC), 2014. [Online]. Available: http://dx.doi.org/ 10.1109/IPEC.2014.6869631

[13] Hongyu Zhu, Donglai Zhang, Bowen Zhang, Zhicheng Zhou, "A nonisolated three-Port DC-DC converter and three-domain control method for PV-battery power systems", IEEE Trans. Industrial Electronics, vol. 62, no. 8. [Online]. Available: http://dx.doi.org/10. 1109/TIE.2015.2393831

[14] M. C. Mira, A. Knott, M. A. E. Andersen, "MOSFET loss evaluation for a low-power stand-alone photovoltaic LED system", IEEE Power Electronics and Drive Systems (PEDS), 2015. [Online]. Available: http://dx.doi.org/10.1109/PEDS.2015.7203489

[15] DATASHEET "BSC050NE2LS OptiMos Power-MOSFET Rev. 2.3", Infineon 2013.

[16] A. Lidow, J. Strydom, M. de Rooij, D. Reusch, GaN Transistors for Efficient Power Conversion 2nd ed. Wiley 2015, ch. 6. [Online]. Available: http://dx.doi.org/10.1002/9781118844779

[17] D. Graovac, M. Purschel, A. Kiep, "MOSFET power losses calculation using the datasheet parameters", Infineon application note 2006. 